\title{
Model of Cloud Video Surveillance Based on Network Coding Cloud Storage System
}

\author{
Qingzhuang Zhao, Kun Li, Hui Li, Yunmin Wang, and Haipeng You
}

\begin{abstract}
Video Surveillance is taking an important role in the daily life and social development, the problem of video's storage is more and more serious as the popular and application of the High Definition Video. We designed a model of video surveillance system based on Network Coding Distributed File System, a storage system that applied Self-repair Code Redundancy Theory, whichi is the most advanced theory in the cloud storge area. While the solution of Converged Network Video Broadcast and Control System for IPTV is integrated so that surveillance video content can be delivered seamlessly on the Internet, then surveillance operators can view video on termnails with different operating system anytime and anywhere. The experimental result demonstrate that system greatly saved storge space compared the traditional video surveillance and improved the flexibility of operating.
\end{abstract}

Index Terms-Cloud storge, IPTV, network coding, video surveillance.

\section{INTRODUCTION}

Video Surveillance System is taking an important role in the daily life [1] and social development, China Telecom have constructed "Global Eye" [2] and China Unicom constructed "Wide View" with different scale throughout the county, with the ultimate goal that construct a Video Surveillance System all over the country similar to the Voice and Data Service Network, which can gain profit by providing service of remote real-time video Surveillance and video playback .

However, three problems must be solved for Video Surveillance System's revolution. Firstly, the operator view video of surveillance anytime and anywhere; Secondly, the video should be displayed on termnals with different operating system, such as Windows, Andriod, iOS and iMAC; Thirdly, low cost and high security of storage for video data should be guaranteed.

Enormous storage space will be need as the content produced all the time. This problem will be more and more serious as the development and spread of High Definition Video [3], display more distinct frame and more clear voice. On the other hand, the number of users is rising sharply, which

Manuscript received March 31, 2014; revised June 12, 2015. This work was supported in part by the National Basic Research Program of China ( 973 Program) under Granted 2012CB315904), the National Natural Science Foundation of China under Granted NSFC61179028, the Basic Research of Shenzhen (No. JCYJ20130331144502026 and No. JCYJ20140417144423192), and the Natural Science Foundation of Guangdong Province under Granted NSFGD S2013020012822.

Qingzhuang Zhao, Hui Li, Yunmin Wang, and Haipeng You are with Shenzhen Graduate School of Peking University, Shenzhen, 518055, China (e-mail: zhaoqz@pku.edu.cn).

Kun Li is with School of Electric Engineering, Beijing Jiaotong University, Beijing, 100044, China (e-mail: likun0203@126.com). also increase the demand of storage space. As a result, the discussion that Video Surveillance System should adopt the solution of Cloud Storage [6] has become a hot topic (see Table I).

\begin{tabular}{|l|l|l|}
\hline \multicolumn{2}{|c|}{ TABLE1: TECHNOLOGY OF CLOUD STORGE } \\
\hline $\begin{array}{l}\text { Technology } \\
\text { Repy Backup }\end{array}$ & $\begin{array}{l}\text { low } \begin{array}{l}\text { low computational } \\
\text { complexity }\end{array} \\
\begin{array}{l}\text { Erasure Code } \\
\text { Redundancy [4] }\end{array}\end{array}$ & $\begin{array}{l}\text { low space usage, } \\
\text { low efficiency } \\
\text { low efficiency }\end{array}$ \\
\hline $\begin{array}{l}\text { Self-repair Code } \\
\text { Redundancy [5] }\end{array}$ & $\begin{array}{l}\text { large repair } \\
\text { bandwidth, high } \\
\text { lomputational } \\
\text { low efficiency, } \\
\text { low repair bandwidth, low } \\
\text { computational complexity, }\end{array}$ \\
& $\begin{array}{l}\text { vary in term of } \\
\text { different between space and } \\
\text { complexity }\end{array}$ \\
\hline
\end{tabular}

Cloud Storage is the development and improvement of Distributed File Storage Technology, the earliest Distributed Storage System [7] stemmed from 1976, Entertainment DEC's File Access Listener (FAL). The technology of Cloud Storage will provide perfect solution for the problems that the Video Surveillance Area is confronted.

High-reliability and high availability is the core competitiveness and advantage of the Cloud Storage, the current technology includes Copy Backup Redundancy and Error Correction Code Redundancy [8]. Of which the technology of Copy Backup Redundancy is studied earliest, and shares the the largest percentage in the market, but high space usage and low efficiency are the shortages. For example, 3 Gigabit space is occupied when 1 Gigabit data is storaged using the technology of Three Copies of the Backup Redundancy.

While the technology of Erasure Code Redundancy has developed for 5 years approximately, there are some Entertainments like Google, Facebook and Taobao of China are engaged in research on it. As a little advanced technology, on one hand it can solve the problem of Copy Backup Redundancy Technology exists, such as high space usage and low efficiency; on the other hand, it causes thorny problems such as large Repair Bandwidth and high Computational Complexity, which would cause high load capacity and instability of system in the environment of big data. As a result, it is common occurrence that large numbers of storage nodes are crashed in a Cloud Storage System Cluster.

We have researched the Technology of Self-repair Code Redundancy [9] for server years, which is the most advanced technology of Cloud Storage, as it can solve both the high space usage, low efficiency of Copy Backup Redundancy and arge Repair Bandwidth, high Computational Complexity of Erasure Code Redundancy [10]. We have applied this 
technology to solve the problems of Video Surveillance, so as our research achievement, Converged Network Video Broadcast and Control Technology for IPTV. We have proposed our solution, IPTV Video Surveillance Based on Network Coding Distributed File System, NCIVS for short. We implemented the model system, and the experiment data demonstrate that our system have many advantages.

The rest of this paper is organized as follows: discussed problems for Video Surveillance in Section II. Our proposed approach converged Network Coding Cloud Storage and IPTV solution for Video Surveillance, NCIVS, is present in Section III, besides the implementation details of the proposed framework is described in Section IV. Section V describes the future work layout. Finally, we conclude the paper in Section VI.

\section{SOLVED PROBLEMS}

The target of NCIVS is to improve traditional Video Surveillance System from server aspects, which is listed as follows.

Firstly, improve scalability and elevate the security of Storage System in contrast with traditional local disk storage's poor scalability and low security, as the record data will be diminished once the node which content is stored is damaged .
Secondly, decrease the cost and investment of the storage system, which increase the days of Video Surveillance can record and support the High Definition Video perfectly.

Thirdly, operators of the surveillance can view the video anytime and anywhere use any devices, that is to say video view are supported cross platform that users can view thorough any terminals with any operating systems, no matter Windows or Andriod [11], Mac Os.

\section{PROPOSED APPROACH}

\section{A. Architecture of System}

A brief framework of NCIVS framework is shown in Fig. 1. In contrast to the traditional Video Surveillance [12], NCIVS have integrated two advantaged technology, one is Network Coding Cloud Storage Technology, as is marked by NCDFS in Fig. 1, and the other is Converged Network Video Broadcast and Control System for IPTV, as is marked by NVBCS in Fig. 1. NCDFS can be deployed everywhere, which is connected with the other parts of system via Internet, so the content is stored not only in the local disk but also on the Internet all over the country even the world. Of course, only the users have license and authority can get the content from Internet immediately and conveniently.

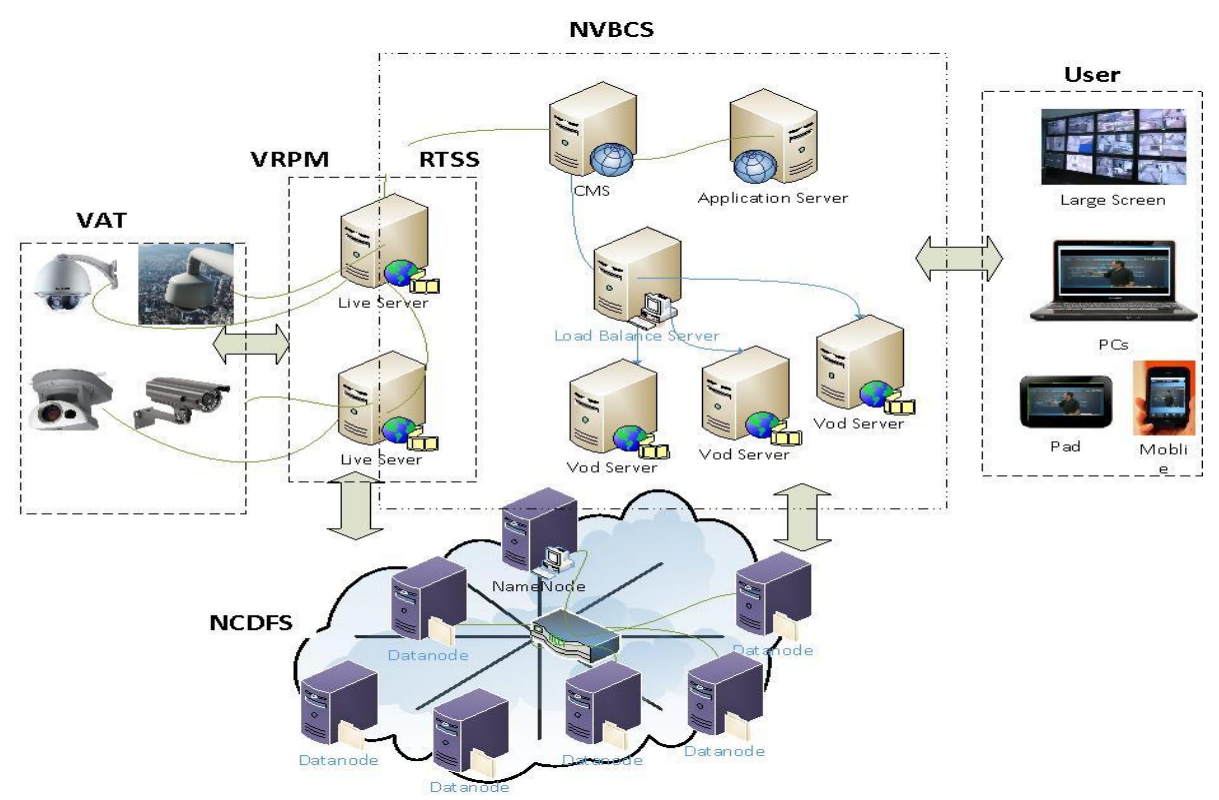

Fig. 1. Framework of the model of cloud video surveillance.

\section{B. System Components}

The componets of NCIVS is described precisely in the following.

Video acquisition terminal(VAT): VAT is used to capture the picture and video from the real world, it may includes many items, such as a mobile camera, a fixed cam or Recording and Broadcasting System, which transmit video signal into VRPM for other process.

Video receiveing and processing module (VRPM): Two main function is provided by the VRPM. On one hand, the video signal that transmited by VAT is transformed into other format so that video can be distributed on the Internet; On the other hand, VRPM support for real time live, it distribute the signal so that users can watch the target in the real time over Internet .

Real time storage system (RTSS): This module is important as it connects video content and Storage System, it writes the video content into Storage System continually for later processing and playbak. As surveillance is continuous, writing into Storage System must be also continuous all the time in a stream format, every a certain period a big video file is produced.

Network coding distribute file system [13] (NCDFS): NCDFS is an implemented Cloud System based on the most advanced theory, Self-repair Code Redundancy Theory, the 
fundamental of which will be described in the Implementation of System and the detail is precisely illustrated in the [8]-[10]. Compareed with Copy Backup Redundancy, NCDFS saves approximately 60 percentage space [14], it means that the system can save approximately 60 percentage investment and 60 percentage electricity consumption everyday, which will be a enormous digital.
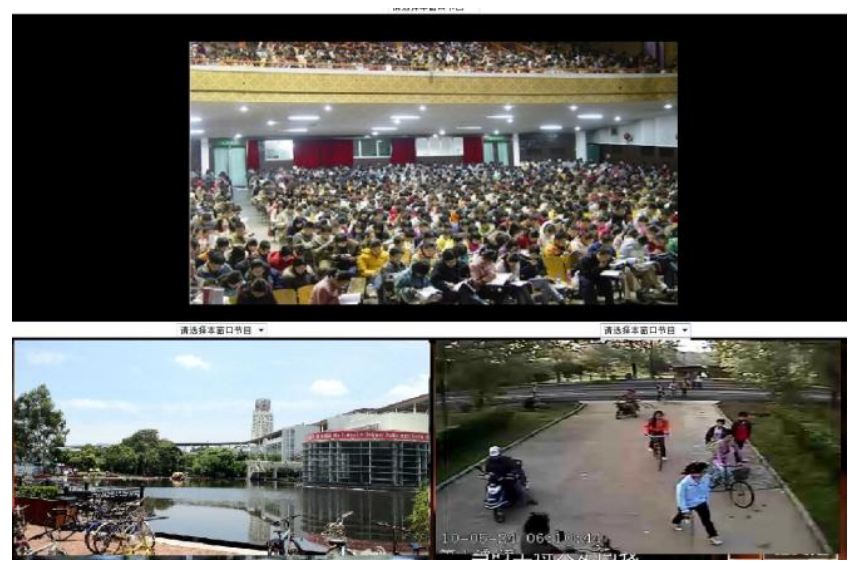

Fig. 2. Capture of three frame watched through Internet.

Network video broadcast and control system (NVBCS): This system is support for view videos or live on the Internet on various terminals without the limit of operating system. And another highlight is self-adaption Bitrate of video stream for the difference of using PC and moble terminals, which is a key issue and will be described in Section III-B precisely.

\section{IMPLEMENTATION OF SYSTEM}

\section{A. Implementation}

We implemented the solution using various technologies and deployed in a local area network. Fig. 3 shows a picture that captured on a screen display three video at the same time through Internet. The current Implementation has four main parts.

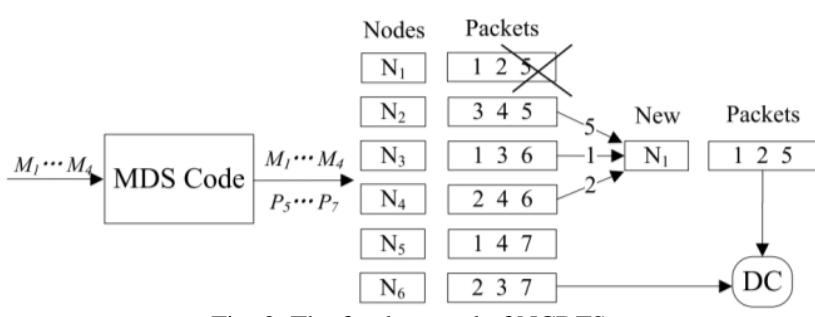

Fig. 3. The fundamental of NCDFS.

- Network Coding Distribute File System is implemented using server code, such as BRS, BMBR, BMSR.

- Web for Network Video Broadcast and Control System, a web page for mana of video content and provide service as vod and live.

- Andriod for users watch on the moble terminals.

- The time shifting storge for video live, the core part of VRPM and RTSS.

\section{B. Key Issues}

There are two key issues in the completion of the system: NCDFS and NVBCS. The fundamental of NCDFS shows in the Fig. 4, we construct our system on Hadoop, an open source cloud storge system. To adapt varied operating system of termnaals, we adopt m3u8 video format. When the video is stored into the Storge System, another m3u8 [15] format of video is generated, which will be transmit to users when the request of moble terminals is responseed.

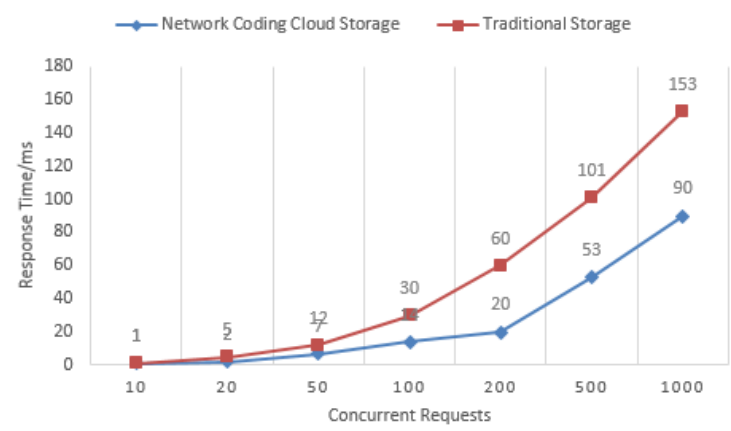

Fig. 4. Experment result,response time as the concurrent requests vary that use different storage system.It demostrate that when the concurrent requests is low, the gap of response time is very small.

\section{FUTURE WORK}

Three directions and aspects of future work is listed as follows. To deal with enormous users visited a video at the same time, P2P [16] should be adopted, as NCDFS will have a long delay when enormous users visited due to its limited read rate. Fortunately, $\mathrm{P} 2 \mathrm{P}$ is a perfect way to make up it when enormous users are emerged. Fault tolerant mechanism should be constructed to response the emergency that network is cut off accidentally. Big Data [17] is more and more important and applied to the Video Surveillance, and have a significant value, such as we can predict emergency and other accidents by processing and analyzing from big data.

\section{EXPERIMENTS}

We deployed two models of video surveillance, one based on trandional cloud storage system, the other based on NCDFS, then, we experiment from two aspects. On one hand, video surveillance based on NCDFS saves approximately 60 percentage space compared with video surveillance based on storage system used Copy Backup Redundancy. On the other hand, the gap of response time used two storage systems is very narrow when the number of concurrent requests in not very high. Though there is a wide gap when the number of concurrent requests rises, the capability of video surveillance based on network coding system is satisfied as there are not high concurrent requests for the video surveillance.

\section{CONCLUSIONS}

We introduced a model of video surveillance, NCIVS, in this paper, described its components and demonstrated its advantages and significant value. We compared NCIVS with traditional video surveillance and optimized video surveillance using traditional cloud storge respect to the cost, stability and security of storge system. Future work directions are considered. Of course, the research on Network Coding Cloud Storge will go on to support more available cloud storge system for video surveillance, and we will focus on the 
$\mathrm{P} 2 \mathrm{P}$ to support massive users online at the same time and big data to make the NCIVS more intelligent.

\section{REFERENCES}

[1] M. A. Hossain, "Cloud-based mobile IPTV terminal for video surveillance," Coll. of Comput. \& Inf. Sci., King Saud Univ., Riyadh, Saudi Arabia, 2014.

[2] J. Jian and F. Yan, "Research of telecom global eye project in a region of Xinjiang on risk identification and evaluation," Chong Qing University of Posts and Telecommunications, 2010.

[3] B. Bennett, "High definition video transcoding for transport over satellite," Defense Inf. Syst. Agency, 2012.

[4] T. A. Courtade, "Optimal allocation of redundancy between packet-level erasure coding and physical-layer channel coding in fading channels," Dept. of Electr. Eng., Univ. of California, Los Angeles, 2011.

[5] T.-H. Wu, "A memory yield improvement scheme combining built-in self-repair and error correction codes," Dept. of Electr. Eng., Nat. Tsing Hua Univ., Hsinchu, Taiwan, 2012.

[6] D. Slamanig, "On cloud storage and the cloud of clouds approach," Inst. for Appl. Inf. Process. \& Commun., 2012.

[7] Q.-Y. Zhu, "The system architecture for the basic information of science and technology experts based on distributed storage and web mining," Fac. of Comput. Eng., Huaiyin Inst. of Technol, 2012.

[8] H.-X. Hou, H. Li, and H.-Y. Zhang, "The application of binary regenerating codes for distributed storge systems," Peking University Shenzhen Graduate School, 2013.

[9] H.-X. Hou, H. Li, and K. W. Shum, "General self-repairing codes for distributed storage systems," Shenzhen Key Lab. of Cloud Comput. Tech. \& App, Peking Univ., 2013.

[10] H.-X. Hou and H. Li, "Practical self-repairing codes for distributed storage," Shenzhen Key Lab. of Cloud Comput. Tech. \& App, Peking Univ., 2012.

[11] R. Guo and T.-S. Zhu, "MobileSens: A framework of behavior logger on Andriod mobile device," Grad. Univ. of Chinese Acad. of Sci., 2011.

[12] D. A. Rodriguez-Silva, L. Adkinson-Orellana, and F. J. Gonz'lez-Castano, "Video surveillance based on cloud storage," Network \& Applic. Dept., Gradiant, Vigo, Spain, 2012.
[13] X.-X. Huang, H.-X. Hou, and H. Li, "Study on binary regenerating codes for big data storge system," Shenzhen Key Lab. of Cloud Comput. Tech. \& App, Peking Univ., 2012.

[14] B.Zhu, H. Li, and H.-X. Hou, "Replication-based distributed storage systems with variable repetition degrees," Shenzhen Key Lab. of Cloud Comput. Tech. \& App, Peking Univ., 2012.

[15] Y. Can et al., "A new mobile streaming system base-on http live Streaming protocol," Sch. of Comput. Sci. \& Eng., South China Univ. of Technol., 2011.

[16] A. Spognardi, A. Lucarelli, and R. D. Pietro, A methodology for P2P file-sharing traffic detection," Dipt. di Informatica, Universita di Roma, 2005.

[17] C.-Q. Ji, Y. Li, W.-M. Qiu et al., "Big data processing in cloud computing environments," Coll. of Inf. Sci. \& Technol, Dalian Maritime Univ., 2012.

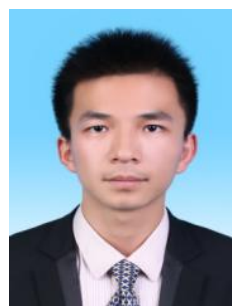

Qingzhuang Zhao was born in 1991. He received his B.E. from Shenzhen Graduate School of Peking University. Currently, he is studying a master's degree of electronic and computer school of course Peking University. Now his research interests include cloud storage, converged network video broadcast and online education. He is a member of Shenzhen Eng. Lab of Converged Network Video Broadcast Technology and he has been worked for the project of Guangdong Educational Cloud.

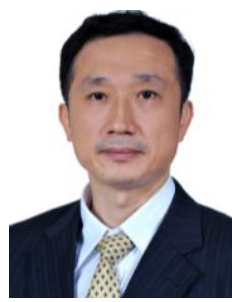

Hui $\mathbf{~ L i ~ w a s ~ b o r n ~ i n ~ 1 9 6 4 . ~ H e ~ i s ~ a ~ p r o f e s s o r ~ o f ~}$ Shenzhen Graduate School of Peking University, and he garduated from the Chinese University of Hongkong with doctor's degree. Now his research interests include cloud storage and big data, network coding theory and distributed file system, converged network video broadcast. He is the dean of Shenzhen Eng. Lab of Converged Network Video Braodcast Technology and the vice-chairman of Cloud Computing Industry Association of Guangdong. 